FETAL ALOCHOL SYNDROME

The relationship between prenatal maternal alcohol use and growth and morphologic abnormalities in the offspring of 650 women studied prospectively is reported from the School of Medicine and Department of Mathematics and Statistics, University of Pittsburgh, PA. Low birth weight, decreased head circumference and length, and an increased rate of fetal alcoholic effects were all found to be significantly correlated with exposure to alcohol during the first two months of the first trimester. The average amounts of alcohol consumed in the first trimester for wamen who were delivered of infants with no, one, or multiple minor physical anomalies were $0.67,0.98$, and 1.07 drinks per day, respectively $(P=0.5)$. The corresponding average daily volumes for mothers of babies exhibiting no, one, or multiple fetal alcohol effects were $0.69,0.85$, and 1.28 $(\mathrm{P}=0.13)$. The time of greatest impact of drinking was in the early part of the first trimester. (Day NL et al. Prenatal exposure to alcohol: Effect on infant growth and morphologic characteristics. Pediatrics September 1989, 84:536-541).

COMMENT. Data from this study have also demonstrated an effect of alcohol use on the sleep electroencephalographic patterns of newborns and on growth at eight months of age. (Scher $M$ et al. Pediatr Res $1988 ; 24: 101)$. This study identifies and emphasizes the importance of exposure to alcohol early in the first trimester as a cause of intrauterine growth retardation and/or morphologic abnormalities in newborn infants. The infant is also at risk if mother drinks alcohol during the breast-feeding period.

\title{
ALOOHOL DURING BREAST-FEEDING AND INFANT DEVELOPMENT
}

The relation of the mother's use of alcohol during breast feeding to the infant's development at one year of age has been investigated in 400 infants studied in the Department of Epidemiology, University of Michigan, Ann Arbor, Department of Epidemiology and Pediatrics, University of Washington, Seattle. Mental development, as measured by the Bayley Mental Development Index, was unrelated to maternal drinking during breast-feeding. However, motor development, as measured by the Psychamotor Development Index, was significantly lower in infants exposed regularly to alcohol in breast milk with a dose response relation. (Little $R E$ et al. Maternal alcohol use during breast-feeding and infant mental and motor development at one year. $\underline{\mathrm{N}}$ Engl J Med Aug 17, 1989; 321:425-30).

COMMENT. Nursing mothers can no longer enjoy a glass of beer as a prescribed method for sedating a sleepless baby wi thout risking a detrimental effect on the infant's development. The practice of offering the parents a complimentary dinner with champagne at the time of discharge from maternity units will have to cease, or if continued, with moderation and caution. It may be argued that the results of this study are suggestive but the detrimental effects on the development are not sufficiently severe to be clinically important for the individual child. The finding that the mental development of infants exposed to alcohol through breast feeding was not affected is of interest and might be explained by the timing of the exposure and the age at which the child was tested. 